Leddis Esther González-Viloria

DOI $10.35381 / \mathrm{cm} . v 6 \mathrm{i} 1.347$

\title{
Aulas Multigrado: Una alternativa de colaboración y cooperación en los espacios educativos
}

\section{Multigrade classrooms: An alternative of collaboration and cooperation in educational spaces}

\author{
Leddis Esther González-Viloria \\ legovis@hotmail.com \\ Institucion Educativa San Juan Bautista de El Retiro, Bolivar \\ Colombia \\ https://orcid.org/0000-0001-8305-372X
}

Recibido: 15 de abril de 2020

Publicado: 30 de junio de 2020

\begin{abstract}
RESUMEN
La investigación tiene como objetivo analizar las Aulas Multigrado como una alternativa de colaboración y cooperación en los espacios educativos de Colombia. Se realizó una revisión documental sobre las aulas multigrado en la escuela rural. El método aplicado es de carácter analítico-descriptivo. Se plantea la condición de preservar lo distintivo de cada entorno, e incluso se considera que no estaría demás, en ciertos casos, ruralizar un poco la escuela urbana, en el sentido de contextualizarla, de hacerla de tamaño más comprimida, con relaciones interpersonales más posibles, con la probabilidad de compatibilizar los espacios y las organizaciones, potenciando una educación más individualizada que beneficie los procesos afectivos y cognitivos.
\end{abstract}

Descriptores: Cooperación educacional; articulación educativa; zona de prioridad educativa; aulas multigrado. (Palabras tomadas del Tesauro UNESCO).

\section{SUMMARY}

The objective of the research was to analyze the Multigrade Classrooms as an alternative of collaboration and cooperation in the educational spaces of Colombia. A documentary review was carried out on the multigrade classrooms in the rural school. The applied method is analytical-descriptive. The condition of preserving the distinctiveness of each environment is proposed, and it is even considered that it would not be unnecessary, in certain cases, to ruralize the urban school a little, in the sense of contextualizing it, making it more compressed in size, with more possible interpersonal relationships, with the probability of making spaces and organizations 
compatible, promoting a more individualized education that benefits affective and cognitive processes.

Descriptors: Educational cooperation; educational articulation; educational priority area; multigrade classrooms. (Words taken from the UNESCO Thesaurus).

\section{INTRODUCCIÓN}

A nivel mundial el mundo educativo está sujeto a profundos cambios socioculturales producto del avance constante de las tecnologías de información y comunicación que involucran todos los aspectos de la vida del hombre. En ese sentido, (Hepp, 2009) expresa que este nuevo modelo de estructura social, donde la globalización de la sociedad y las nuevas tecnologías de la información y comunicación se han unido buscando con ello que las personas no solo le den utilidad sino que puedan disfrutar de las bondades que tienen las mismas ya que son consideradas las herramientas del futuro.

Es por ello que el sector educativo no debe divorciarse de las mismas frente a los acelerados cambios del conocimiento, y las necesidades de saber en diversas organizaciones de la sociedad. Por tanto para (Martínez y Bustos, 2011) indica que todo este escenario viene afectando a los diversos sistemas educativos del siglo XXI, el cual tiene sus particularidades dificultades y posibilidades que son producto de las transformaciones producidas en los contextos rurales y que han llevado a dar paso a una nueva identidad frente a una sociedad compleja y variada.

En el mismo orden de ideas (Aróstegui y Martínez, 2008) expresa que todos estos cambios no necesariamente van referidos al contexto pedagógico en si sino que abarcan otros factores entre ellos el demográfico ideológico económico entre otros en el marco de un escenario que refleja una manera de entender la equidad como la mera aplicación de estrategias y líneas de actuación iguales para todos los contextos, propia de un pensamiento neoliberal y neoconservador que no distingue territorios.

En ese sentido la escuela rural está limitada por una forma errónea de ver la educación como forma de controlar los procesos muy por el contrario debe verse frente a la consecución de logros y en la competitividad planteada por un modelo de escuela 
estandarizada en la que prevalece la homogeneidad. En relación a lo expuesto Rojas, (2006) opina que la diversidad y la heterogeneidad como notas de identidad de la escuela rural se convierten entonces en un reto para la mejora, unidas a la existencia de condiciones socioculturales heterogéneas y de contextos y territorios rurales.

De igual modo, (Bernat, 2009) expresa que la escuela rural tiene como objetivo central formar personas libres con conciencia de su situación responsables de su actuar y que luchen por convertirse en protagonistas en la historia de su emancipación. Sólo así podremos plantear las posibles aportaciones de la escuela rural multigrado al logro de una escuela de calidad, partiendo de la convicción de que sus características diferenciadoras contribuyen a este logro.

\section{El aula multigrado en la escuela rural}

Refiere (Abós et al. 2015) que en Colombia el sistema educativo es variado en las distintas comunidades sobre todo en las rurales ya que cada una tiene sus políticas educativas propias en cuanto a su forma de actuar. Por tanto los tipos de agrupamientos viene clasificados desde diversas (Centros Rurales Agrupados, Zonas Escolares Rurales, Colegios Públicos Rurales, entre otros). Más sin embargo, la concepción entre aulas multigrados y escuelas rural no difiere en las diversas comunidades colombianas.

La multigraduación es el componente principal de las escuelas rurales o de aulas multigrado entendiendo la misma como aquella donde existe un solo docente como figura principal que atenderá a estudiantes de diferentes edades y conocimientos en la cual comparten un mismo espacio educativo.

Sobre el particular, (Little, 2011) expresa que existe una variedad de acepciones en las cuales se pueden mencionar clases combinadas con cursos mezclados de manera forzada y con estudiantes de diferentes edades es decir refiere que las mismas son creadas por una necesidad educativa más no está comprobada su intención pedagógica como tal, esto se evidencia en el contexto rural colombiano. Mientras que otros términos como "agrupamientos verticales", "no-graduadas" se suelen relacionar con aulas multigrado creadas como opción pedagógica en escuelas no rurales y 
rurales. Es así que, independientemente de la intención que ellas traen; su función no es más que atender diversos estudiantes con características educativas y edades diferentes centrándose en el valor pedagógico que ellas representan, donde existe una figura de tutor o guía el cual dirige los procesos que allí se generan.

A partir de estos señalamientos (Santos, 2011) la importancia de una didáctica multigrado va dirigida a la utilización de estrategias didácticas en las cuales se organice el espacio, el tiempo los recursos y los aspectos curriculares de tal manera que puedan generarse un aprendizaje verdaderamente significativo dejando a un lado la didáctica psicologizada, epistemologizada y prescriptiva, como nos alerta.

En estos escenarios educativos la razón fundamental del proceso pedagógico es poder convivir estudiantes de diferentes edades y cursos y así complementarse unos a otros sin que la edad sea un impedimento; es decir aquí es valedero el principio de que todos aprendemos de todos sin que se tome en cuenta su momento evolutivo, sino enfatizar en un aprendizaje colaborativo y el contacto directo con contenidos de diversos niveles educativo.

Este fenómeno específico, referido al enriquecimiento académico interedad en aulas multigrado, ha aparecido mencionado durante los últimos años con diferentes denominaciones, pero con significados complementarios: teoría de los círculos concéntricos de aprendizaje (Boix, 1995), aprendizaje por contagio (Bustos, 2010) o circulación de saberes (Santos, 2011).

El hecho de compartir un mismo espacio educativo, de interaccionar de forma natural, ya va a representar poder atender y reflexionar contenidos diferentes en cuanto a grados y edades, conceptos y descripciones que vienen a remarcar la impregnación mutua en el fenómeno asimétrico, expandido y fluctuante de los procesos de aprendizaje de alumnado de diferentes grados en la misma aula de clases.

De la misma manera, para Siegel y Shaughnessy (2004 p.564):

el mayor error en la enseñanza durante los pasados siglos ha sido tratar a todos los niños como si fueran variante del mismo individuo, y de este modo encontrar la justificación para enseñarles las mismas cosas de la misma manera, 
Por tanto, no se tiene en cuenta que cada uno tiene sus particularidades y que pueden representar un recurso valioso dentro del proceso. Así también para (Mulryan-Kyne, 2014) la filosofía de estas aulas multigrados es que permite al docente una integralidad en los procesos y no solo aplicar una estrategia didáctica es aquí donde el docente conoce de manera detallada a sus estudiantes puesto que las características particulares así lo determinan.

Aquí en estos espacios se genera un aprendizaje colaborativo en sus prácticas didácticas asi como en las diversas formas y maneras de aprender ; más sin embargo señala (Quail y Smyth, 2014) es necesario la asertividad de los docentes en las estrategias a emplear más allá de las prefijadas aquí es importante tomar en cuenta las experiencias externas del grupo a pesar de la diferencia de edades, todo ello va a generar el reordenamiento del espacio tiempo recursos que le permitan incentivar a investigar y creer en sí mismos como constructores de su propio aprendizaje.

Aquí se pone en práctica la metodología activa-participativa la cual va centrada en el trabajo integral e interrelacionado de conocimientos en el aula, promocionando la autonomía y el aprendizaje colaborativo entre sus miembros también la adquisición de competencias transversales y metodológicas así como la adquisición de capacidades de autogestión del aprendizaje confianza personal sobre todo en la toma de decisiones y aprehensión de relaciones inter e intra dependencia personal, sin depender de otras personas.

Sin embargo, (Ribchester y Edwards, 2008) indican que aquí en estas aulas el docente tiende a confundir autonomía con trabajo individual, aunque se puede considerar que el estudiante es autónomo cuando es capaz de trabajar solo sin la ayuda del tutor ; aunque no todas las veces ese nivel de autonomía supone la posible pérdida de la misma si se involucra en una relación más estrecha de actividad colaborativa.

En el mismo orden de ideas aquí el trabajo colaborativo va a conducir como estimulante para que cada estudiante asuma responsabilidades que son las que sumadas van a garantizar el trabajo en equipo y asi poder dar respuestas a los diferentes objetivos o planes preestablecidos. 
Asimismo, (Mulryan-Kyne, 2014) en estas escuelas se genera la independencia entre sus estudiantes a su vez que los estudiantes desarrollan sus habilidades interpersonales; acá el aprendizaje se da por contagio entre las diferentes edades puesto que existe un acompañamiento entre todos los miembros del grupo, esto contribuye a elevar tanto su autoestima como el desarrollo social de los estudiantes. En este escenario educativo el estudiante se convierte en tutor o guía del resto desarrollando así sus capacidades metacognitivas, aquí ellos toman conciencia y valoran el aprendizaje pudendo compensar dicho proceso con sus experiencias, aquí se aprende a aprender o en su defecto aprender haciendo.

Por su parte, (Santos y Martínez, 2011) con relación a lo anterior expresan los autores que para que se genere el proceso de enseñanza deben existir dos aspectos o componentes fundamentales los recursos y las actividades de aprendizaje. En cuanto a las actividades de aprendizaje son las que el docente de escuelas rurales debe hacer mayor énfasis puesto que representan la adquisición de competencias, es decir son los ejes de planificación curricular, en la mayoría de los casos más que las estrategias didácticas.

Las actividades de aprendizaje son el componente con el que suelen reflexionar en primer lugar para su encaje posterior en la estrategia didáctica. A pesar de que los docentes consideran importante y necesario el desarrollo deductivo de la planificación curricular partiendo de las competencias para llegar a la evaluación. En este proceso para el docente rural la actividad de aprendizaje vendría a ser la razón principal a programar y la más valorada de forma creativa e innovadora en todos los procesos de enseñanza aprendizaje.

\section{El mito de la mala calidad educativa en las aulas multigrado}

En relación a ello, (Alpe y Fauguet, 2010) indican que aún no existen pruebas que corroboren el falso mito de la mala calidad académica de estas escuelas multigrados ellos alegan que el fracaso escolar en el ámbito rural viene dado por las condiciones en la que se encuentran aglomerados los estudiantes y esto hace que el ambiente y la situación se vuelva caótica y anacrónica. 
En cuanto a ello (Feu y Gelis 2009, p. 10) la cataloga como "enseñanza circular o concéntrica" sobre este particular los estudiantes mayores una vez que se les esté explicando a los más pequeños, esas lecciones le sirven de repaso, sirviéndole los mismos para aplicarlos posteriormente. (Alpe, 2015) en estos espacios los estudiantes mayores sirven como guías o tutores a los de menor edad lo que repercute en varios aspectos sobre todo en su autoestima, autonomía, responsabilidad, madurez de sus conocimientos, representa una gran satisfacción para todos los involucrados en cuanto a la aplicabilidad de las respectivas estrategias de aprendizaje. En el mismo orden de ideas, (Feu y Gelis, 2009) señalan que es importante acabar con el mito de la mala calidad de estas escuelas rurales, por el contrario es necesario resaltar las ventajas de las mismas según varios aportes dados por docentes sobre la pregunta generada "¿de qué puede presumir la escuela rural?".

Entre las ventajas se pueden mencionar : aquí en estas escuelas se desarrolla permanentemente un proceso de experimentación educativa facilitando una pedagogía activa, además de que los docentes son integrales y polivalentes, los estudiantes son heterogéneos, aquí el docente se puede dedicar de manera individualizada a cada estudiante haciendo que la calidad de aprendizaje d estos estudiantes, aquí tanto el currículo como el horario de trabajo es flexible, a pesar de tener poco espacio físico, el mismo puede ser reinventado y ajustado según las necesidades, también se aplica y prevalece el respeto por encima de cualquier cosa, asimismo el sistema de evaluación se hace de manera integral haciendo que los estudiantes se vinculen con el entorno.

\section{Desmitificando la mala calidad educativa y revelando la riqueza de la educación rural}

En palabras de (Martín-Moreno Cerrillo 2012) al realizar una revisión bibliográfica sobre estas escuelas llega a la conclusión de que por ser reducido el tamaño de los mismos no se considera una limitación, por el contrario representa una virtud, de hecho las investigaciones así lo han dejado ver y han manifestado que este tipo de organización se consideran más eficaces y efectivas que el resto de las instituciones 
Pittman y Haughwout (1987) señalan que existe menos índice de fracaso escolar en estas escuelas y que el nivel de satisfacción de los estudiantes es superior al de las escuelas grandes.

- Lee Smith (1995) manifiesta que aquí en estas escuelas los estudiantes aprenden mucho más y con mejor calidad.

- Stochkard y Mayberry (1992) ellos manifiestan que en estas escuelas los problemas de conducta son mínimos debido a las particularidades de las mismas.

Meier (1996) desglosa siete razones que apoyan el mayor éxito de las escuelas pequeñas:

- El sentido de pertenencia sobre todo en lo que respecta al contexto comunitario

- El trato respetuoso entre docente y estudiante haciendo que sus relaciones sean más cordiales.

- Transparencia en los procesos administrativos evitando la burocracia en los procesos y posibilitando la individualización.

- La seguridad en estos espacios es importante debido a que cualquier situación indebida se detecta rápidamente.

- El acercamiento y participación escuela comunidad hace que los resultados sean los mejores.

- Debe existir un control permanente para así evitar procesos burocráticos indebidos y con ello poder mostrar el rendimiento tanto de estudiantes como de docentes.

- Fácil control para los procesos administrativos y académicos en función al número de docentes al momento sobre todo de expresar sus opiniones y así poder obtener mejores relaciones interpersonales.

En función de ello es importante destacar que el tamaño no influye en su totalidad a garantía de la misma, así como tampoco el éxito, pero si es considerado un factor de interés en el logro de los objetivos planificados. Martín-Moreno Cerrillo (2002) expone 
algunas ventajas de estas escuelas en cuanto a estudiantes, docentes, y organización del entorno de aprendizaje.

\section{a) En cuanto a los docentes:}

Los docentes tienden a ser más coherentes y significativos en cuanto a el enfoque pedagógico a emplear.

Se establecen relaciones interpersonales de mayor calidad por la particularidad de las mismas.

Se observan un docente más proactivo hacia la labor que desempeña al igual que la cooperación y solidaridad con su equipo de trabajo.

Los docentes muestran mayor tendencia a integrar áreas curriculares 0 interdisciplinares.

\section{b) En relación a los estudiantes:}

La instrucción recibida es más individualizada.

Se percibe mayor entusiasmo al momento de realizar las actividades escolares.

Se demuestra el trabajo en equipo con mayor frecuencia en la realización de actividades, lo que realza la cooperación y solidaridad como valores fundamentales. La autoestima de cada uno de ellos tanto personal como académico tiende a ser más efectivo.

Se perciben menos situaciones de indisciplina ni conductas agresivas, así como tampoco el consumo de sustancias estupefacientes, robos, entre otros.

\section{c) En relación a la organización del entorno del aprendizaje:}

Prevalece el desarrollo de actividades de forma individual en los estudiantes en función de que los grupos son reducidos y con diferencias de edades y multinivel.

En estas aulas los horarios de trabajo académico se pueden flexibilizar debido a las características propias que ellas generan. En cuanto a los entornos de aprendizaje se consideran más ordenados y seguros. También se visualiza un mayor enlace entre las culturas de los estudiantes con las personas adultas. Por su parte un mayor énfasis en la relación del aprendizaje escolar con el entorno. 


\section{METODOLOGÍA}

En cuanto al tipo de investigación, esta investigación se clasifica como analítico descriptivo, ya que se contrasta una serie de eventos con la teoría existente y se recolecta información que permite el direccionamiento y medios empleados; asimismo, Tamayo (2009), indica que es descriptiva ya que comprende la descripción, registro, análisis e interpretación de la naturaleza actual, composición o procesos de los fenómenos. En cuanto al tipo analítico Hurtado (2010, citado por Baleta 2020) señala que la investigación analítica tiene como objetivo analizar un evento y comprenderlo en términos de sus aspectos menos evidentes incluyendo tanto el análisis como la síntesis. Es decir, implica la interpretación de lo analizado en función de criterios descritos, considerando los objetivos en estudio.

En el mismo sentido se plante con un diseño documental; el cual se estará revisando la bibliografía y apoyar la formación de un profesional dentro de lo que hoy se conoce como sociedad del conocimiento, donde se le imparte una formación prudente, pero le abre una nueva visión para impulsar el aprender a lo largo de su gestión.

En esta investigación, se realiza una revisión documental sobre las aulas multigrado. La misma tiene como objetivo presentar las aulas que agrupan alumnado de distintos niveles, pudiendo abarcar las "unitarias" que tienen los alumnos de todos los niveles existentes con un único maestro y las "graduadas incompletas" que pueden tener varios niveles (con uno o varios ciclos) y donde en cada clase hay un maestro.

\section{DISCUSIÓN Y REFLEXIONES}

En cuanto a las actividades escolares, se tienen varios aspectos que debe ser mejorados en cuanto a rutinas escolares se refiere, es decir llega un momento en que los estudiantes del aula multigrado se vuelven autónomos por estar el docente atendiendo el resto de los otros grados. En la mayoría de los casos los estudiantes lo hacen de forma espontánea sin ningún tipo de argumento ni programación por parte del docente. 
En ese sentido estos espacios educativos permiten que los estudiantes gestionen su propio proceso de aprendizaje de manera individual puesto que en el desarrollo del mismo se descubren un sinnúmero de competencias que ponen de manifiesto.

Es de resaltar que existen de igual modo el proceso de adaptación ajustado a las competencias de cada estudiante.

Otra particularidad de estas aulas es el aprendizaje colaborativo entre estudiantes de diferentes edades y las buenas relaciones interpersonales que se desarrollan algunas monitoreadas por el docente y otras de manera espontánea.

Las actividades a desarrollaren estos espacios se hace de manera general y luego es tarea del docente adaptarlos a los diferentes grados según sea la necesidad y realidad, mientras que otras se pueden trabajar de manera conjunta.

El libro de textos para estos tipos de aulas representa uno de los recursos principales sobre todo cuando existen actividades comunes para varios grados, a pesar de que algunos lo llevan a un plano secundario. Es el docente el encargado de planificar este tipo de estrategias y llevarlas al aula de clases.

Del mismo modo en estos espacios el material impreso contribuye a reforzar las estrategias en el aula lo cual complementa los textos escritos en cuanto a la realidad circundante y la multigraduacion; sin embargo los docentes manifiestan la necesidad de que deberían existir otro tipo de recursos para poder alternar las diferentes estrategias, esto con la finalidad de poder recompensar el esfuerzo de algunos estudiantes al cumplir con los horarios y actividades asignadas, de manera de evitar cualquier conducta indebida.

\section{REFERENCIAS CONSULTADAS}

Abós, P. et al. (2015). La escuela rural y la política educativa española. Diferencias entre Comunidades Autónomas: Andalucía, Aragón y Cataluña. International Studies on Law and Education, 19, 73-90.

Alpe, Y. (2015) "Quels sont les déterminants de la réussite dans la trajectoire des éléves des milieux ruraux?". En Café pedagogique 2005. https://n9.cl/dtjar

Alpe, Y. y Fauget, J.L. (2010). "Lécole rurale, école de modernité?" Consultado en Google el 25 de octubre de 2010 en Observatoire delécole rurale. 
Aróstegui, J. L. y Martínez, J. B. (2008). Globalización, posmodernidad y educación. La calidad como coartada neoliberal. Madrid: Akal-UNIA

Baleta Araujo, E. (2019). Transferencia de conocimiento como estrategia gerencial del sector farmacéutico en Colombia. Revista Arbitrada Interdisciplinaria Koinonía, 4(8), 148-165. http://dx.doi.org/10.35381/r.k.v4i8.275

Bernat, A. (2009). Escuela rural e ideologías. Jornadas sobre educación en el medio rural: Encrucijadas y respuestas. DVD. Teruel: Universidad de Zaragoza.

Boix, R. (1995). Estrategias y recursos didácticos en la escuela rural. Barcelona: Graó.

Bustos, A. (2010). Aproximación a las aulas de escuela rural: heterogeneidad y aprendizaje en los grupos multigrado. Revista de Educación, 352, 353-378.

Feu I Gelis, J. (2009) "La escuela rural en España: apuntes sobre las potencialidades pedagógicas, relacionales y humanas de la misma". Revista Digital eRural, Educación, cultura y desarrollo rural. Año 2, no 3 . Junio. Disponible en: http://educacion.upa.cl/revistaerural/erural.htm.

Hepp, A.; (2009) "Herkunfts-, Ethno- und Weltorientierte: Aneignungstypen der kulturellen Identität und kommunikativen Vernetzung in der Diaspora". Medien \& Kommunikationswissenschaft 58 (3), pp. 320-342.

Hurtado, J. (2010). El proyecto de investigación. Sypal. Caracas. Venezuela

Little, A. (2011). Multigrade teaching: towards an international research and policy agenda. International Journal of Educational Development, 21, 481-497.

Martínez, J. B. y Bustos, A. (2011). Globalización, nuevas ruralidades y escuelas. Profesorado. Revista de currículo y formación del profesorado, 15(2), pp. 3-12. Disponible en: http://www. ugr.es/ recfpro/rev152ed.pdf

Martín-Moreno Cerrillo, Q. (2012) "Claves para la calidad de los centros educativos rurales". En Lorenzo Delgado, M y otros (2012). Liderazgo educativo y escuela rural. Vol. II. Granada. Grupo Editorial Universitario.

Mulryan-Kyne, C. (2014). Teaching and learning in multigrade classrooms: what teachers say. The Irish Journal of Education, 35, 5-19.

Pittman, R B. y Haughwout, P (1987): "Influence of High School Size on Dropout Rate”. En Educational Evaluation and Policy Analysis, vol. 9, no4, Winter, pp. 337-343 
Quail, A. y Smyth, E. (2014). Multigrade teaching and age composition of the class: The influence on academic and social outcomes among students. Teaching and Teacher Education, 43, 8090.

Ribchester, C. Edwards, B. (2008). Co-operation in the countryside: Small primary school clusters. Educational Studies, 24(3), 281-293.

Santos, L. (2011). Aulas multigrado y circulación de los saberes: especificidades didácticas de la escuela rural. Profesorado. Revista de curriculum y formación del profesorado, 15(2), 71-91. Siegel.

Siegel, J. y Shaughnessy, M. (1994). Educating for understanding: A conversation with Howard Gardner. Phi Delta Kappan, 75(7), 564-578.

Smith, L. (1995). Comprensión de la lectura. Análisis psicolingüístico de la lectura y su aprendizaje. México: Trillas.

Stochkard, J.; Mayberry, M. (1992): Effective educational environments. Newbury Park, C.A.C. Corwin.

Tamayo, M. (2009). Metodología formal de la investigación científica. México: Limusa/Noriega. 